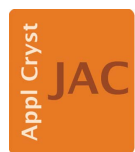

JOURNAL OF APPLIED CRYSTALLOGRAPHY

ISSN 1600-5767

Keywords: call for papers; neutron scattering; instrumentation

\section{Call for papers on Advanced Neutron Scattering Instrumentation}

Journal of Applied Crystallography invites papers on advanced neutron scattering instrumentation to appear in a special issue of the journal that will be published approximately in April 2018.

Neutron scattering facilities are called to address an ever-expanding mission in the investigation, development and application of a broad range of materials: from investigating cultural heritage artifacts to advanced bio-materials, from studies of phase transition and quantum matter to advanced engineering composites for aircraft. This breadth of applications, together with the complex problems they present for making scientific and technical progress, are key drivers for new advanced neutron sources and novel instrumentation that covers enormously broad scale ranges in both time and space.

This special issue on Advanced Neutron Scattering Instrumentation aims to highlight innovative advances in neutron scattering instrumentation at facilities around the world that address the broad scientific and industrial scope of applications that these facilities are called to meet. The scope of papers includes instrumentation at recent and planned facilities as well as instrument upgrades and new instrument development concepts. The emphasis of the volume will be innovative instrumentation and new scientific capabilities.

The deadline for paper submission will be 1 September 2017, with final publication of the special volume planned for April 2018 to coincide with the 50th anniversary of Journal of Applied Crystallography. This volume will be edited by Guest Editors Dimitri Argyriou (Ames Lab) and Andrew Allen (NIST), together with with several Guest Coeditors. Provisionally, these will include Ken Herwig (SNS), Kenji Nakajima (JPARC), Dan Neumann (NIST) and Masa Arai (ESS).

For enquiries on this volume, especially from potential authors regarding proposed paper content, please feel free to contact Dimitri Argyriou (argyriou@ameslab.gov) or Andrew Allen (andrew.allen@nist.gov). Completed articles should be submitted only via the special issue submission page at http://submission.iucr.org/submit/j?Qconf=ansi. 